\title{
(Conformal) Killing vectors in the Newman-Penrose formalism
}

\author{
Garry Ludwig* $\quad$ S. Brian Edgar ${ }^{\dagger}$
}

June 7,2001

\begin{abstract}
Finding (conformal) Killing vectors of a given metric can be a difficult task. This paper presents an efficient technique for finding Killing, homothetic, or even proper conformal Killing vectors in the NewmanPenrose (NP) formalism. Leaning on, and extending, results previously derived in the GHP formalism we show that the (conformal) Killing equations can be replaced by a set of equations involving the commutators of the Lie derivative with the four NP differential operators applied to the four coordinates.

It is crucial that these operators refer to a preferred tetrad relative to the (conformal) Killing vectors, a notion to be defined. The equations can then be readily solved for the Lie derivative of the coordinates, i.e. for the components of the (conformal) Killing vectors. Some of these equations become trivial if some coordinates are chosen intrinsically (where possible), i.e. if they are somehow tied to the Riemann tensor and its covariant derivatives.

If part of the tetrad, i.e. part of null directions and gauge, can be defined intrinsically then that part is generally preferred relative to any Killing vector. This is also true relative to a homothetic vector or a proper conformal Killing vector provided we make a further restriction on that intrinsic part of the tetrad. If because of null isotropy or gauge isotropy, where part of the tetrad cannot even in principle be defined

\footnotetext{
${ }^{*}$ Department of Mathematical Sciences, University of Alberta, Edmonton, Alberta, Canada T6G 1S6

${ }^{\dagger}$ Department of Mathematics, University of Linköping, Linköping, Sweden S-581 83.
} 
intrinsically, the tetrad is defined only up to (usually) one null rotation parameter and/or a gauge factor, then the NP-Lie equations become slightly more involved and must be solved for the Lie derivative of the null rotation parameter and/or of the gauge factor as well. However, the general method remains the same and is still much more efficient than conventional methods.

Several explicit examples are given to illustrate the method.

\section{Introduction}

Within tetrad formalisms there is a standard procedure for finding (conformal) Killing vectors ((C)KVs), i.e. Killing vectors (KVs), a homothetic vector $(\mathrm{HV})$, and/or proper conformal Killing vectors (CKVs) for a given spacetime or for specifying their existence while solving Einstein's equations for the spacetime. This procedure, which includes integration of the tetrad version of the (conformal) Killing equations, is, however, long and complicated as well as inefficient due to several redundancies in the equations.

Recently [1, 2], we have developed a new method for obtaining an HV and/or KVs for spacetime solutions found in the Geroch-Held-Penrose 3 (GHP) formalism. We proved that the (conformal) Killing equations can be replaced as key equations by a set of sixteen commutator equations involving the four GHP differential operators and a new generalized Lie derivative operator (called the GHP Lie derivative). This procedure can, with some care, be extended to CKVs as well.

In this paper we adapt our method to the better-known, if more cumbersome, Newman-Penrose 4 (NP) formalism. We shall replace the (conformal) Killing equations by a set of sixteen commutator equations involving the four NP derivative operators and the ordinary Lie derivative. In principle, to find $\mathrm{HV}, \mathrm{KVs}$ and CKVs we only need to construct an appropriate null tetrad for a given metric. However, the calculations can often be shortened considerably if we know something about the geometric nature of the tetrad and the coordinates used. We usually have such knowledge if we are in the process of finding a spacetime solution possessing certain symmetries. But even for a given metric we may calculate, for example, the Weyl tensor, and use one or both of its principal null directions as the tetrad's null directions. As we shall see, such knowledge, though not necessary in principle, usually reduces significantly the complexity of the new equations. 
The main aim of this paper is to address the problem of finding $(\mathrm{C}) \mathrm{KVs}$, i.e. an HV, KVs, and CKVs, using the NP formalism, and to illustrate our method with explicit examples.

One of the main differences between the NP and GHP formalisms is that the former uses specific null tetrads whereas the latter deals only with classes of null tetrads (called GHP tetrads here), where only the directions of the two real null vectors are fixed but not the gauge. The gauge freedom consists of the ability to make spin- and boost transformations.

In a previous paper [2] we defined the notion of an intrinsic GHP tetrad, as well as that of a preferred GHP tetrad relative to a vector, in particular relative to a (C)KV. We argued that an intrinsic GHP tetrad was preferred relative to any KV. (For an HV or a CKV the situation is a bit more complicated). We proved the equivalence of the usual (conformal) Killing equations with the GHP-Lie commutator equations, equations involving the commutators of a newly defined generalized Lie derivative, called the GHP Lie derivative, with the four GHP differential operators corresponding to a preferred GHP tetrad, applied to the four coordinates.

In this paper, we define the notion of a preferred gauge relative to a $(\mathrm{C}) \mathrm{KV}$ as one in which the GHP Lie derivative and the ordinary Lie derivative coincide. As we shall see, such a gauge always exists. The (conformal) Killing equations can then be replaced by commutator equations involving commutators of the ordinary Lie derivative and the NP differential operators applied to the four coordinates, provided that the tetrad is preferred relative to the $(\mathrm{C}) \mathrm{KV}$ involved.

The question is then how to obtain such a preferred tetrad, especially when the metric possesses more than one (C)KV and we want to find all of them at once. One way is to leave enough arbitrariness in the tetrad so that the commutator equations can choose a tetrad appropriate to each $(\mathrm{C}) \mathrm{KV}$. However, that may complicate the equations somewhat ${ }^{\mathrm{F}}$. For KV it is usually possible (and most efficient) to find an intrinsic tetrad, i.e. one that can be defined in terms of the Riemann tensor and its covariant derivatives. Such a tetrad is then generally preferred relative to any KV. In case of null isotropy or gauge isotropy we may have to be satisfied with a partially intrinsic tetrad. If we want to include an $\mathrm{HV}$ or a CKV in our search, it is again best to ensure (where possible) that the tetrad is intrinsic. However,

\footnotetext{
${ }^{1}$ This is not necessarily so. There was no such complication in the example of the pure radiation metric of Ref.[2].
} 
in addition, the conditions defining the null directions and the gauge must be properly conformally weighted. For an HV, it suffices if there is a proper conformal weight relative to a constant conformal transformation.

In section 2, we review the GHP approach to KVs and HV, as developed in Ref.[2], and extend the results to CKVs. In section 3 we adapt these results to the NP formalism. Several important lemmas will lead to the key theorem involving the NP-Lie commutator equations. In section 4, we discuss and illustrate our method further, with examples of metrics quoted in canonical tetrads. Some appropriate remarks in section 5 will conclude the paper.

The NP version of our method is deduced in section 3 from our earlier GHP version[2]. Although we could have obtained these NP results independently and entirely in the NP formalism it was much quicker to modify the GHP results. However, the basic theorems at the end of Section 3, and the theorems we deduce and use in Section 4 can be understood without any detailed knowledge of the GHP formalism.

One of our motivations for this work is to be able to apply our results to the invariant/Karlhede classification (IC) program[5] for spacetimes. In that program there exist algorithms to determine respectively the dimension of the isometry group [6] of the spacetime and to determine whether the

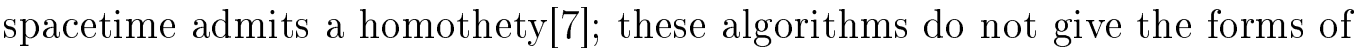
the symmetry groups, nor the explicit symmetry vectors. Our intention is to fill this gap in a subsequent paper, by developing our method to find an HV and KVs explicitly and efficiently from the information supplied by the IC of a metric. We shall therefore use notation and conventions in this paper which are consistent with the IC program.

There are a number of general results [8, 9] which give restrictions on a $\mathrm{CKV}$; in particular, it is known that the only vacuum spacetimes admitting a CKV are the plane waves. Such theorems can be used to simplify our calculations in specific applications.

Today there are a number of computer packages which calculate symmetries in given spacetimes. It might be thought that these would replace existing methods and, indeed, any calculations done by hand. However, it should be recognized that these packages are still relatively limited. For instance, recently, one package was unable to obtain results directly, results pertaining to some symmetries of the Kerr metric[10], even though, as pointed out in Ref. [8], these particular symmetries were known not to exist as a consequence of some general theorems. On the other hand, in section 4 of the present paper, our methods establish directly and quickly the non-existence of an HV 
and CKVs for the Kerr-Newman metric. It is our intention eventually to apply our methods not only to the classification and calculation of symmetries of existing metrics but to use these methods as an efficient means of imposing symmetries in a coordinate-invariant manner in the search for new solutions.

\section{Preferred GHP tetrads and the GHP-Lie com- mutator equations}

Kolassis and Ludwig[1] introduced a generalized Lie derivative $L_{\xi}$ which is defined like the ordinary Lie derivative $£_{\xi}$ except that ordinary derivatives are replaced by covariant derivatives. Equivalently,

$$
L_{\xi}=£_{\xi}-\xi^{\mu}\left(p \zeta_{\mu}+q \bar{\zeta}_{\mu}\right),
$$

where $(p, q)$ are the GHP weights of the quantity operated upon, and

$$
\zeta_{\mu}=\gamma l_{\mu}+\varepsilon n_{\mu}-\alpha m_{\mu}-\beta \bar{m}_{\mu},
$$

where $\mathbf{l}, \mathbf{n}, \mathbf{m}, \overline{\mathbf{m}}$ are null tetrad vectors (normalized by $l_{\mu} n^{\mu}=1, m_{\mu} \bar{m}^{\mu}=$ -1 , other inner products zero), and $\alpha, \beta, \gamma, \varepsilon$ are the "bad" Newman-Penrose spin-coefficients that disappear from the GHP formalism.

A more useful operator turned out to be a further generalization, namely

$$
\mathrm{E}_{\xi}=L_{\xi}-\frac{p}{4}\left(\mathcal{P}-\mathcal{P}^{\prime}+\mathcal{P}^{*}-\mathcal{P}^{*}\right)-\frac{q}{4}\left(\mathcal{P}-\mathcal{P}^{\prime}+\mathcal{P}^{*}-\mathcal{P}^{*}\right),
$$

where

$$
\mathcal{P}=n_{\mu} L_{\xi} l^{\mu}
$$

and $\mathcal{P}^{\prime}, \mathcal{P}^{*}$, and $\mathcal{P}^{\prime *}$ are its three companions 11], i.e. its images under the prime, the star, and the prime-star operations. An equivalent definition of this GHP Lie derivative is ${ }^{\mathrm{F}} \mathrm{F}$

$$
\begin{aligned}
£_{\xi} & =£_{\xi}+\left(\frac{p+q}{4}\right)\left(l_{\mu} £_{\xi} n^{\mu}-n_{\mu} £_{\xi} l^{\mu}\right) \\
& +\left(\frac{p-q}{4}\right)\left(\bar{m}_{\mu} £_{\xi} m^{\mu}-m_{\mu} £_{\xi} \bar{m}^{\mu}\right) .
\end{aligned}
$$

\footnotetext{
${ }^{2} \mathrm{E}_{\xi}$ as defined here reduces to that of Eq.(4) of Ref.[2] only in the case of a KV. This should have been made more explicit there.
} 
Let us define the remaining tetrad components $\mathcal{Q}$ and $\mathcal{R}$ of $E_{\xi} l^{\mu}$ by

$$
\mathrm{E}_{\xi} l^{\mu}=\frac{1}{2}\left(\mathcal{P}+\mathcal{P}^{\prime}\right) l^{\mu}+\mathcal{R} n^{\mu}-\mathcal{Q} m^{\mu}-\overline{\mathcal{Q}} \bar{m}^{\mu},
$$

and similarly for their companions under the the prime, star and star-prime operations. A GHP tetrad is said to be preferred ${ }^{\mathrm{F}} \mathrm{P}$ relative to the vector $\xi$ provided that

$$
\mathcal{Q}^{\prime}+\mathcal{Q}^{*}=0=\mathcal{Q}+\mathcal{Q}^{*}
$$

Such a GHP tetrad always exists but is not unique; it allows for null rotations with parameters $y$ and $z$ satisfying

$$
\mathrm{E}_{\xi} y=0=\mathrm{Ł}_{\xi} z .
$$

If, in addition, the (conformal) Killing equations hold, namely

$$
\begin{aligned}
& \mathcal{P}^{\prime}=-\mathcal{P}-\varphi, \quad \mathcal{P}^{*}=-\mathcal{P}^{*}-\varphi \\
& \mathcal{Q}=\mathcal{Q}^{\prime *}, \quad \mathcal{Q}^{\prime}=\mathcal{Q}^{*} \\
& \mathcal{R}=\mathcal{R}^{\prime}=\mathcal{R}^{*}=\mathcal{R}^{\prime *}=0
\end{aligned}
$$

then

$$
\mathrm{E}_{\xi} l^{\mu}=-\frac{1}{2} \varphi l^{\mu},
$$

and similarly for its companions. A tetrad satisfying Eqs.(10) (including the companions) is said to be GHP Lie recurrent (GLR).

Note again that the $(\mathrm{C}) \mathrm{KV}$ is an $\mathrm{HV}$ if the function $\varphi$ is a constant and a $\mathrm{KV}$ if $\varphi$ is zero; otherwise it is a CKV.

Although in the above we defined the notion of preferred null directions, i.e. of a preferred GHP tetrad, for an arbitrary vector $\xi$, its main usefulness is relative to a $(\mathrm{C}) \mathrm{KV}$. When the null directions are preferred relative to a (C) KV, the generalized Lie derivative of such a GHP tetrad then takes its simplest form, given by Eq.(10) and companion equations. In particular, relative to a KV, a preferred GHP tetrad gets annihilated by the GHP Lie derivative $€$. We say it is GHP Lie derived.

\footnotetext{
${ }^{3}$ The motivation for this definition is not entirely obvious here. For an explanation the reader is referred to Ref.[2].
} 
More generally, for a (C)KV $\xi$ and corresponding conformal factor $\varphi$, if a quantity $\eta$ satisfies

$$
\left(\mathrm{E}_{\xi}-w \frac{\varphi}{2}\right) \eta=0,
$$

for some number $w$, we say that it is $G L R$ (relative to $\xi$ and with associated factor $\varphi$ ) and that it has $w$-weight $w$. It is not hard to show that if $\eta$ is properly conformally weighted then the $w$-weight coincides with the conformal weight. For the contravariant tetrad vectors and for spin-coefficients well-behaved in the conformally extended GHP formalism 12, $w=-1$; for Weyl tensor tetrad components, $w=-2$. If, again with $\xi$ a (C)KV and $\varphi$ the corresponding conformal factor, a quantity $\eta$ satisfies

$$
\left(£_{\xi}-w \frac{\varphi}{2}\right) \eta=0
$$

we say that $\eta$ is (ordinary) Lie recurrent (relative to the vector $\xi$ and with $w$-weight $w$ ).

\section{Definition 1.}

The GHP scalars with respect to a GHP tetrad are the well-behaved GHP spin-coefficients, all the Riemann tensor tetrad components, together with all properly GHP weighted combinations of these and their GHP derivatives.

In Ref.|2] we proved the following lemmas.

Lemma 1 If the GHP tetrad is GLR relative to a vector $\xi$ and with associated factor $\varphi$ then $\xi$ is a $(C) K V$ with $\varphi$ the conformal factor, and the GHP tetrad is necessarily preferred relative to $\xi$.

Lemma 2 If $\xi$ is a $(C) K V$ with conformal factor $\varphi$ then the GHP tetrad is GLR relative to $\xi$ and with associated factor $\varphi$ iff it is preferred relative to $\xi$.

Lemma 3 If the GHP tetrad is preferred relative to a vector $\xi$ then it is GLR relative to $\xi$ with associated factor $\varphi$ iff $\xi$ is a (C)KV with conformal factor $\varphi$. 
Lemma 4 Given an $H V$ or a $K V \xi$, all properly $G H P$ weighted combinations of the GHP tetrad, the GHP scalars, and the tetrad components of $\xi$ itself are $G L R$ relative to $\xi$ provided that the tetrad is preferred relative to $\xi$.

Lemma 5 If the GHP tetrad is GLR relative to a vector $\xi$ then there exists a particular gauge in which the tetrad is (ordinary) Lie recurrent relative to $\xi$.

Lemma 6 Relative to an $H V$ or a $K V$, all GHP scalars of type (0,0) are (ordinary) Lie recurrent in an arbitrary gauge of a preferred GHP tetrad.

Note that the last two lemmas are already in a form suitable for the NP formalism.

We now wish to generalize Lemma 4 to include proper conformal Killing vectors, i.e. CKVs. We know from Eqs.(27)-(35) and their companions in Ref.|2] that most GHP scalars which are $G L R$ relative to an $\mathrm{HV}$ or a KV in a tetrad preferred with respect to that $\mathrm{HV}$ or $\mathrm{KV}$, are also $G L R$ relative to a CKV in a tetrad preferred with respect to that CKV, e.g., $\left(\in_{\xi}+\frac{1}{2} \varphi\right) \kappa=0$. However, there are some GHP scalars which are not $G L R$ relative to a CKV. For example, $\left(\mathrm{E}_{\xi}+\frac{1}{2} \varphi\right) \bar{\pi}=\frac{1}{2} \partial \varphi$ and $\left(\mathrm{E}_{\xi}+\frac{1}{2} \varphi\right) \tau=-\frac{1}{2} \partial \varphi$. It follows easily that $(\bar{\pi}+\tau)$ is $G L R$ since $\left(E_{\xi}+\frac{1}{2} \varphi\right)(\bar{\pi}+\tau)=0$. Similarly, we can show that all properly conformally weighted GHP scalars are $G L R$.

So we can deduce the general result,

Lemma 7 Given a $(C) K V \xi$, all properly conformally weighted and properly GHP weighted combinations of the GHP tetrad, the GHP scalars and the tetrad components of $\xi$ itself are GLR relative to $\xi$, provided that the GHP tetrad is preferred relative to $\xi$.

To be explicit 12, the properly conformally weighted spin coefficients (all with conformal weight -1 ) are, $\kappa, \sigma, \lambda, \nu, \rho-\bar{\rho}, \mu-\bar{\mu}, \tau+\bar{\pi}$. The tetrad components of the Weyl tensor are, of course, properly conformally weighted (all with conformal weight -2). Although the Ricci tensor tetrad components by themselves are not, the following combinations are properly conformally weighted (with conformal weight -2 ),

$$
\Phi_{00}+\frac{1}{2}\left[-\mathrm{p}(\rho+\bar{\rho})+\frac{1}{2}(\rho+\bar{\rho})^{2}+\kappa(\pi-\bar{\tau})+\bar{\kappa}(\bar{\pi}-\tau)\right] \text { (and companions), }
$$




$$
\begin{aligned}
& \Phi_{01}+\frac{1}{2}\left[\mathrm{~b}(\bar{\pi}-\tau)+\frac{1}{2}(\bar{\pi}+\tau)(\rho+\bar{\rho})+\kappa(\mu+\bar{\mu})\right] \text { (and companions), } \\
& \Phi_{11}+\frac{1}{4}\left[\mathrm{~b} \mu-\mathrm{b}^{\prime} \rho+\partial \pi+\bar{\partial} \bar{\pi}-\frac{1}{2}(\tau-\bar{\pi})(\bar{\tau}-\pi)+\frac{1}{2}(\rho+\bar{\rho})(\mu+\bar{\mu})\right] \\
& \Lambda+\frac{1}{4}\left[\mathrm{p}^{\prime} \rho-\mathrm{p} \mu+\partial \pi+\bar{\gamma} \bar{\pi}+\frac{1}{2}(\rho+\bar{\rho})(\mu+\bar{\mu})+\frac{1}{2}(\tau-\bar{\pi})(\bar{\tau}-\pi)\right] .
\end{aligned}
$$

To avoid misunderstandings, we point out that all GHP scalars could be described as 'properly conformally weighted', provided that the conformal factor is constant, i.e. for an $H V$ or a $K V$. However, when we use the phrase 'properly conformally weighted' we will be referring to CKVs, for which the conformal factor is a non-constant function.

Key to our approach in the GHP formalism is the following

Theorem 8 In a preferred GHP tetrad relative to a $(C) K V \xi$, with $\varphi$ the conformal factor,

$$
\left[p, E_{\xi}\right] \eta=\frac{\varphi}{2} p \eta+\frac{p+q}{4} \eta p \varphi
$$

and its companion equations hold for any $(p, q)$-weighted scalar. Conversely, if Eqs.113) (including companions) hold for four functionally independent $(0,0)$-weighted quantities and for the same function $\varphi$ throughout, then $\xi$ is a (C) KV with conformal factor $\varphi$, and the GHP tetrad is necessarily preferred relative to $\xi$.

When attempting to find preferred null directions relative to a number of (C) KV, it is often very helpful to look for intrinsically defined null directions such as the principal null directions of the Weyl tensor. Like any intrinsic quantity, these are fixed in terms of the Riemann tensor and its covariant derivatives to whichever order is necessary. Such an intrinsic GHP tetrad exists for most spacetimes but not for all. In practice, unless the spacetime is conformally flat, the $\mathbf{l}$ - direction of the null tetrad can be taken to be a principal null direction (p.n.d.) of the Weyl tensor; it is then intrinsic by definition. If there is a second p.n.d. it can serve as the $\mathbf{n}$ - direction, giving us an intrinsic GHP tetrad. It was shown in Ref. [2] that an intrinsic GHP tetrad is preferred relative to any KV. In fact, a close look at the proof shows that when the tetrad's null directions are principal null directions of the Weyl tensor (or if they are otherwise intrinsically fixed in a proper conformally weighted manner), this is so even for an $\mathrm{HV}$ or a CKV. In type $\mathrm{N}$, in the case of vanishing spin-coefficient $\rho$, it may still be possible to fix the $\mathbf{n}$ - direction in terms of the Riemann tensor but such an intrinsic $\mathbf{n}$ direction is not necessarily preferred relative to a CKV. 
Worse perhaps, there are metrics for which it is not possible to fix the second null direction intrinsically, and we then have null direction isotropy. But it should be remembered that relative to a (C)KV, there always does exist a preferred $\mathbf{n}$ - direction irrespective of whether there exists an intrinsic one. Since we then may not know explicitly what that preferred null direction is, we have to leave some arbitrariness in the GHP operators of the GHP-Lie commutator equations (13). The GHP tetrad used is now defined only up to an unknown null rotation parameter. The equations become a bit more involved; nevertheless it is usually still quite easy to solve them. Along with the Lie derivatives of the coordinates, i.e. the components of the $(\mathrm{C}) \mathrm{KV}$, we now obtain the Lie derivative of the null rotation parameter. If that turns out to be zero then, in hindsight, the null rotation parameter could have been taken to be zero (since a preferred GHP tetrad is defined only up to Eqs.(8)).

When we are unable to fix the $\mathbf{n}$ - direction intrinsically, an alternative method is to use the formalism of Machado and Vickers [13]; but then it becomes necessary to define a new generalized Lie derivative operator and to determine the generalizations of Lemmas 4 and 7 and of Theorem 8 to that formalism.

\section{Preferred tetrads and the NP-Lie commuta- tor equations}

While the GHP approach to finding (C)KV is our favourite, the NP formalism is still much more popular than the GHP one. For this reason we now adapt our technique to the NP formalism.

Let us start with a tetrad where the null directions have been fixed somehow. There still remains the two-dimensional gauge freedom

$$
\widehat{l}^{\mu}=A^{2} l^{\mu}, \widehat{n}^{\mu}=A^{-2} n^{\mu}, \widehat{m}^{\mu}=\exp \left(i \alpha_{0}\right) m^{\mu} .
$$

To obtain a specific gauge and hence a specific tetrad, we need to fix the gauge factor $A \exp \left(\frac{i}{2} \alpha_{0}\right)$. 


\section{Definition 2.}

We define a preferred gauge relative to a vector $\xi$ to be one for which

$$
£_{\xi} \eta=£_{\xi} \eta
$$

for any quantity $\eta$.

Lemma 9 There always exists a preferred gauge relative to a vector $\xi$.

Proof. For $(0,0)$ - quantities, Eq.(15) holds in any gauge. Since any other quantity of interest here is obtained by contracting a tensor with the tetrad it suffices to show that there is a gauge for which

$$
\mathrm{E}_{\xi} l^{\mu}=£_{\xi} l^{\mu}
$$

and companions hold.

$>$ From Eqs.(11),(3) and 4 we have

$$
\begin{aligned}
£_{\xi} l^{\mu} & =£_{\xi} l^{\mu}-\xi^{\nu}\left(\zeta_{\nu}+\bar{\zeta}_{\nu}\right) l^{\mu}-\frac{1}{2}\left(\mathcal{P}-\mathcal{P}^{\prime}\right) l^{\mu} \\
£_{\xi} n^{\mu} & =£_{\xi} n^{\mu}+\xi^{\nu}\left(\zeta_{\nu}+\bar{\zeta}_{\nu}\right) n^{\mu}+\frac{1}{2}\left(\mathcal{P}-\mathcal{P}^{\prime}\right) n^{\mu} \\
£_{\xi} m^{\mu} & =£_{\xi} m^{\mu}-\xi^{\nu}\left(\zeta_{\nu}-\bar{\zeta}_{\nu}\right) m^{\mu}-\frac{1}{2}\left(\mathcal{P}^{*}-\mathcal{P}^{\prime *}\right) m^{\mu}
\end{aligned}
$$

It is not hard to show that if we choose $A$ and $\alpha_{0}$ such that

$$
\begin{aligned}
2 £_{\xi} \ln A & =-\xi^{\nu}\left(\zeta_{\nu}+\bar{\zeta}_{\nu}\right)-\frac{1}{2}\left(\mathcal{P}-\mathcal{P}^{\prime}\right) \\
i \exp \left(i \alpha_{0}\right) £_{\xi} \alpha_{0} & =-\xi^{\nu}\left(\zeta_{\nu}-\bar{\zeta}_{\nu}\right)-\frac{1}{2}\left(\mathcal{P}^{*}-\mathcal{P}^{\prime *}\right),
\end{aligned}
$$

something we can clearly always do, then in the new gauge given by Eqs.(14), Eqs.(16) (including companions) hold.

Note that a preferred gauge is not unique. It is determined only up to boost - rotations whose parameters satisfy

$$
£_{\xi} A=0=£_{\xi} \alpha_{0} .
$$


We now wish to combine preferred null directions (i.e., GHP tetrads) with preferred gauges. Naturally, we make the following

\section{Definition 3.}

A preferred tetrad relative to a vector $\xi$ is one for which both the null directions and the gauge are preferred relative to $\xi$.

We are now in a position to prove the following lemmas.

Lemma 10 A preferred tetrad relative to a vector $\xi$ always exists.

Proof. We showed in Ref.[1] that preferred null directions relative to a vector $\xi$ always exist and in Lemma 9 that a preferred gauge relative to $\xi$ always exists as well.

Lemma 11 If the tetrad is Lie recurrent relative to a vector $\xi$ and with associated factor $\varphi$ then $\xi$ is a $(C) K V$ with $\varphi$ the associated conformal factor, and the tetrad is necessarily preferred relative to $\xi$.

Proof. Let us first note that if a tetrad is Lie recurrent then the corresponding GHP tetrad, given by Eq.(14) for an arbitrary gauge factor, satisfies

$$
\mathrm{E}_{\xi} \widehat{l}^{\mu}=-\frac{1}{2} \varphi \widehat{l}^{\mu}
$$

with similar equations for its companions. This is shown by basically doing the reverse of what was done in the proof of Lemma 9. By Lemma 1 and Eqs.(20) (including companions), $\xi$ is a (C)KV with conformal factor $\varphi$, and the null directions are preferred. Since, in the original gauge, $\mathrm{E}_{\xi}$ becomes $£_{\xi}$, this gauge is preferred, and hence so is the tetrad.

Lemma 12 If $\xi$ is a (C) $K V$ with conformal factor $\varphi$ then the tetrad is Lie recurrent relative to $\xi$ and with associated factor $\varphi$ iff it is preferred relative to $\xi$.

Proof. Suppose that $\xi$ is a (C)KV. If we assume that the tetrad is preferred relative to $\xi$ it follows from Lemma 1 that Eq.(20) and its companions are satisfied. In the given preferred gauge $\ell_{\xi}$ becomes $£_{\xi}$, showing that the tetrad is Lie recurrent.

Conversely, if we assume that the tetrad is Lie recurrent, then by Lemma 11 it is necessarily preferred. 
Lemma 13 If the tetrad is preferred relative to a vector $\xi$ then it is Lie recurrent relative to $\xi$ with associated factor $\varphi$ iff $\xi$ is a $(C) K V$ with conformal factor $\varphi$.

Proof. Suppose the tetrad is preferred relative to a vector $\xi$. Then the corresponding GHP tetrad is preferred. If the tetrad is Lie recurrent then, as in the proof of Lemma 11, the corresponding GHP tetrad is GLR. It follows from Lemma 3 that $\xi$ is a $(\mathrm{C}) \mathrm{KV}$.

Conversely, if $\xi$ is a (C) KV then, since the tetrad is preferred, it follows also from Lemma 3 that the GHP tetrad is GLR. In the preferred gauge this means that the given tetrad is Lie recurrent.

\section{Definition 4.}

The NP scalars with respect to an NP tetrad are the NP spin coefficients, all the Riemann tensor tetrad components, together with all combinations of these and their NP derivatives.

Next, we come to the main results of this paper, namely the NP versions of Lemma 7 and Theorem 8 . We first show

Lemma 14 (a) Given an $H V$ or a $K V \xi$, all combinations of the tetrad, the NP scalars, and the tetrad components of $\xi$ itself are Lie recurrent with respect to $\xi$ provided that the tetrad is preferred relative to $\xi$.

(b) Given a $(C) K V \xi$, all properly conformally weighted combinations of the tetrad, the NP scalars, and the tetrad components of $\xi$ itself are Lie recurrent with respect to $\xi$ provided that the tetrad is preferred relative to $\xi$.

Proof. The proofs of (a) and (b) follow immediately from Lemmas 4 and 7 respectively since, in a preferred gauge, $£_{\xi}=£_{\xi}$. Strictly speaking, these proofs only apply to combinations which are of proper GHP weight; but we can extend the proofs, by direct calculation, to all combinations of the tetrad and NP scalars.

For reference, we will quote all conformally weighted NP scalars. They are the conformally weighted GHP scalars together with $\varepsilon-\bar{\varepsilon}, \gamma-\bar{\gamma}, \alpha+\bar{\beta}$ and all conformally weighted combinations thereof.

Theorem 15 In a preferred tetrad relative to a $(C) K V \xi$, with $\varphi$ the conformal factor,

$$
\left[D, £_{\xi}\right] \eta=\frac{\varphi}{2} D \eta
$$


and its companion equations hold for any scalar quantity $\eta$. Conversely, if Eqs. (21) (including companions) hold for four functionally independent quantities and for the same function $\varphi$ throughout, then $\xi$ is a (C)KV with conformal factor $\varphi$, and the tetrad is necessarily preferred relative to $\xi$.

Proof. Suppose that $\xi$ is a $(\mathrm{C}) \mathrm{KV}$ and that the tetrad is preferred relative to $\xi$. Since the corresponding GHP tetrad is preferred relative to $\xi$, it follows from Theorem 8 that

$$
\left[\mathrm{p}, \mathrm{七}_{\xi}\right] \eta=\frac{\varphi}{2} \mathrm{p} \eta
$$

and companion equations hold. Since the gauge is also preferred relative to $\xi$, Eq.(22) reduces to Eq.(21) for the given preferred tetrad, and similarly for the companion equations.

Conversely, if Eq.(21) and its companions hold in some tetrad for four functionally independent quantities then, for the corresponding GHP tetrad, Eq.(22) and its companions hold. But, by Theorem 8, this means that $\xi$ is a $(\mathrm{C}) \mathrm{KV}$ and the GHP tetrad is necessarily preferred. Since the gauge is preferred iff $€_{\xi}=£_{\xi}$ it follows that the tetrad is preferred.

We have noted at the end of the last section that in order to find preferred tetrad directions (i.e. preferred GHP tetrads) it is often useful to look for intrinsically defined null directions, which can be shown to be preferred for all KV. Furthermore, it can be shown that intrinsically defined null directions which are defined in a proper conformally weighted manner (usually in terms of the Weyl tensor) are preferred with respect to all (C)KV. The principal null directions are particularly useful in this regard.

Similarly, in order to find a preferred gauge, it is often useful to determine if an intrinsic restriction determines the gauge; such a gauge will be preferred for all KV. In order to find a gauge which is preferred with respect to all (C)KVs, it is useful to look at a gauge defined both intrinsically and in a proper conformally weighted manner (which often, but not necessarily, means defined with respect to the Weyl tensor). Such a gauge can be shown to be preferred as follows.

We must show that, for such a gauge, $£_{\xi} \eta=£_{\xi} \eta$ for all GHP scalars $\eta$. It suffices to show this for one $(p, q)$ - weighted quantity $R$ with $p \neq \pm q$. Suppose that the gauge is fixed by the condition $R=1$, where $R$ is intrinsic. For this to be a properly conformally weighted condition, the conformal weight of $R$ must be zero, i.e the $w$ - weight of $R$ must vanish. But then $€_{\xi} R=0=£_{\xi} R$. 


\section{Metrics in Canonical Tetrads}

Because of Theorem 15, our approach to finding (C)KVs is as follows. In a preferred tetrad relative to the $(\mathrm{C}) \mathrm{KV}$ to be found we apply Eq.(21) and its companions to the four coordinates $x^{i}$ and solve these equations for $£_{\xi} x^{i}$ $(i=1,2,3,4)$, thereby obtaining the components of the $(\mathrm{C}) \mathrm{KV}$. If we have succeeded in choosing a tetrad which is preferred relative to a number of different $(\mathrm{C}) \mathrm{KVs}$, then this solution will yield all such (C)KVs.

In order to illustrate simply how our results can be exploited in practice we will now restrict our consideration to metrics which have been constructed following standard routines via an NP tetrad. In principle, we know that we can investigate the symmetries of such metrics via Theorem 15. Obviously, if the NP tetrad is a preferred tetrad then we just apply the theorem directly. Unfortunately, the concept of a 'preferred tetrad' is not used explicitly in the standard NP routines, and so our first task will be to look in some detail at how NP tetrads are constructed, and how they relate to our concept of a 'preferred tetrad'. Fortunately, the manner in which NP tetrads are often chosen - at least partly determined by the Weyl or Ricci (equivalently energy-momentum) tensor and their covariant derivatives - enables us to find these relations explicitly.

A standard NP approach to determining a metric, whose Weyl tensor is required to be of a particular Petrov type, involves adopting the canonical null tetrad associated with that particular Petrov type as the NP tetrad for the calculations. In order for us to exploit such results, we need to know how close the canonical null tetrad of each Petrov type is to a preferred tetrad.

So, in the following practical applications to specific metrics already quoted in canonical NP tetrads, our approach will be first to determine the Petrov type which will tell us to what extent the NP canonical tetrad is related to a preferred tetrad. Once we have transformed the given NP canonical tetrad into a preferred tetrad by a suitable transformation, then we can apply Theorem 15 and use the Lie-NP commutators to determine KVs, HVs and CKVs appropriately. However, in order for our calculations to be as efficient as possible, it is important that we also fully exploit Lemma 14. Since the coordinates to which we apply the Lie-NP commutators are frequently defined in terms of the NP scalars, exploiting the simple recurrence properties of this lemma can considerably simplify the calculations involving the commutators. 


\subsection{PETROV TYPE I with a canonical tetrad}

A canonical null tetrad ${ }^{\mathrm{F}}$ for Petrov type $\mathrm{I}$ is defined by

$\Psi_{1}=0=\Psi_{3}, \Psi_{0}=\Psi_{4}, \Psi_{2} \neq 0$.

Since the tetrad directions as well as the gauge are defined by the Weyl tensor in a proper conformally weighted manner, the tetrad is easily seen to be preferred for all (C)KVs. So we can immediately apply Theorem 15. Being able to decide whether the coordinates of the metric under investigation are Lie recurrent is important for carrying out our calculations with the NP-Lie commutators in the most efficient manner. Lemma 14 gives us the crucial result for the scalars associated with the preferred tetrad. In this case, the canonical tetrad is preferred. Therefore, we can take the conclusions of Lemma 14 completely across to the canonical NP scalars associated with the canonical NP tetrad; hence those coordinates which have been defined from the canonical NP scalars of the canonical NP tetrad are Lie recurrent. We have established the following

Theorem 16 If a given metric is of Petrov I and if an NP tetrad for this metric is in the above canonical form, then all (C)KVs can be found by applying $\left[D, £_{\xi}\right]=\frac{1}{2} \varphi D$ and its companion equations to the four coordinates $x^{i}$, and solving for $£_{\xi} x^{i}$ and the conformal function $\varphi$. Furthermore, (a) all proper conformally weighted combinations of the NP scalars are Lie recurrent relative to each $(C) K V$, (b) all combinations of the $N P$ scalars are Lie recurrent relative to each $K V$ and $H V$.

\section{Example 1.}

The Bell-Szekeres 14 metric, in coordinates $(u, v, x, y)$ is given by

$d s^{2}=2 \exp \left(\frac{U}{2}\left(a^{2}-1\right)\right) d u d v-2 \exp (U(1-a)) d x^{2}-2 \exp (U(1+a)) d y^{2}$

\footnotetext{
${ }^{4}$ It should be noted that there are alternatives for a canonical tetrad of type I spaces, e.g. $\Psi_{0}=0=\Psi_{4}, \Psi_{1}=\Psi_{3}, \Psi_{2} \neq 0$; but we have used the alternative employed in the IC program[5].
} 
where $U=\ln (u+v)$. For a tetrad we may take

$$
\begin{aligned}
l^{\alpha} & =\left(\exp \left(\frac{U}{4}\left(1-a^{2}\right)\right), 0,0,0\right) \\
n^{\alpha} & =\left(0, \exp \left(\frac{U}{4}\left(1-a^{2}\right)\right), 0,0\right) \\
m^{\alpha} & =\left(0,0, \frac{1}{2} \exp \left(\frac{U}{2}(a-1)\right), \frac{i}{2} \exp \left(-\frac{U}{2}(a+1)\right)\right) .
\end{aligned}
$$

Therefore,

$$
\begin{aligned}
& D u=\exp \left(\frac{U}{4}\left(1-a^{2}\right)\right) \quad D v=0 \quad D x=0 \quad D y=0 \\
& \Delta u=0 \quad \Delta v=\exp \left(\frac{U}{4}\left(1-a^{2}\right)\right) \quad \Delta x=0 \quad \Delta y=0 \\
& \delta u=0 \quad \delta v=0 \quad \delta x=\frac{1}{2} \exp \left(\frac{U}{2}(a-1)\right) \quad \delta y=\frac{i}{2} \exp \left(-\frac{U}{2}(a+1)\right) .
\end{aligned}
$$

The spin coefficients are

$$
\begin{aligned}
& \kappa=\nu=\tau=\pi=\alpha=\beta=0 \\
& \lambda=-\frac{a}{2} D U=-\sigma \quad \mu=\frac{1}{2} D U=-\rho \quad \varepsilon=\frac{a^{2}-1}{8} D U=-\gamma,
\end{aligned}
$$

where

$$
D U=\frac{1}{u+v} \exp \left(\frac{U}{4}\left(1-a^{2}\right)\right)=\exp \left(-\frac{U}{4}\left(3+a^{2}\right)\right) .
$$

The tetrad components of the Riemann tensor are

$$
\begin{aligned}
\Phi_{00} & =\Phi_{01}=\Phi_{02}=\Phi_{12}=\Phi_{11}=\Phi_{22}=\Lambda=\Psi_{1}=\Psi_{3}=0, \\
\Psi_{0} & =\frac{a}{4}\left(1-a^{2}\right) \exp \left(-\frac{U}{2}\left(3+a^{2}\right)\right)=\Psi_{4}, \\
\Psi_{2} & =\frac{a^{2}-1}{4} \exp \left(-\frac{U}{2}\left(3+a^{2}\right)\right) .
\end{aligned}
$$


We assume that $a \neq 0$ (otherwise the metric is type D). We also assume that $a \neq \pm 1$ since otherwise the metric is flat. The metric is then vacuum, of Petrov type I. The tetrad is determined completely by the Weyl tensor, and the gauges are defined via properly conformally weighted conditions $\left(\Psi_{0}=\Psi_{4}\right)$. We can therefore apply Theorem 16. But since the metric is vacuum we already know [15] that no CKVs exist, so we need only consider $\varphi$ constant and use part (a).

Although we can use Theorem 16 (a) to determine the Lie recurrence relations for the NP scalars, we are not able to find simple explicit recurrence relations for any of the coordinates. However, if, instead, we focus on $u+v$ and $u-v$, we can use the result that $£_{\xi} \Psi_{2}=-\varphi \Psi_{2}$ to get $£_{\xi}(u+v)=$ $\frac{2 \varphi}{3+a^{2}}(u+v)$. The commutator equations on $(u-v)$ integrate to give

$$
£_{\xi}(u-v)=\frac{2 \varphi}{3+a^{2}}(u-v)+c_{1},
$$

where $c_{1}$ is an integration constant. Hence,

$$
\begin{aligned}
& £_{\xi} u=\frac{c_{1}}{2}+\frac{2 \varphi}{3+a^{2}} u \\
& £_{\xi} v=-\frac{c_{1}}{2}+\frac{2 \varphi}{3+a^{2}} v .
\end{aligned}
$$

The NP-Lie commutator equations on $x$ and $y$ integrate to give, respectively,

$$
\begin{aligned}
& £_{\xi} x=\frac{\varphi}{2\left(3+a^{2}\right)}(1+a)^{2} x+c_{2} \\
& £_{\xi} y=\frac{\varphi}{2\left(3+a^{2}\right)}(1-a)^{2} y+c_{3} .
\end{aligned}
$$

Therefore,

$$
\begin{aligned}
\xi & =\frac{2 \varphi}{3+a^{2}}\left[(u+v) \frac{\partial}{\partial(u+v)}+(u-v) \frac{\partial}{\partial(u-v)}+\frac{x}{4}(1+a)^{2} \frac{\partial}{\partial x}+\frac{y}{4}(1-a)^{2} \frac{\partial}{\partial y}\right] \\
& +c_{1} \frac{\partial}{\partial(u-v)}+c_{2} \frac{\partial}{\partial x}+c_{3} \frac{\partial}{\partial y}
\end{aligned}
$$

The KVs are $\frac{\partial}{\partial u}-\frac{\partial}{\partial v}, \frac{\partial}{\partial x}$, and $\frac{\partial}{\partial y}$. The HV is

$$
4 u \frac{\partial}{\partial u}+4 v \frac{\partial}{\partial v}+(1+a)^{2} x \frac{\partial}{\partial x}+(1-a)^{2} y \frac{\partial}{\partial y}
$$




\subsection{PETROV TYPE II with a canonical tetrad}

A canonical tetrad for Petrov type II is defined ${ }^{\mathrm{F}}$ ए by $\Psi_{4}=1, \Psi_{2} \neq 0$, all other $\Psi$ equal to zero.

In Petrov type II the canonical tetrads are fixed completely by the Weyl tensor, and it is easy to see that such a tetrad is a preferred tetrad for any $\mathrm{KV}$ that may exist in the spacetime. Therefore, we can immediately apply Theorem 15 to investigate KVs. However, the above canonical tetrad will not be preferred for CKVs, or even for an $\mathrm{HV}$, since the standard gauge specialization $\left(\Psi_{4}=1\right)$ is not a proper conformally weighted condition.

Therefore, in Petrov type II, although a canonical tetrad is sufficient to investigate $\mathrm{KVs}$, to become a preferred tetrad with reference to an $\mathrm{HV}$ or a $\mathrm{CKV}$, it will require a gauge transformation. To achieve this we simply introduce a gauge parameter $A \exp \left(\frac{i}{2} \alpha_{0}\right)=\left(\Psi_{4} / \Psi_{2}\right)^{\frac{1}{4}}$, so that in the transformed tetrad, $\Psi_{4}=\Psi_{2}$, and this gauge specialization is now preferred. Therefore, for the transformed tetrad we can apply Theorem 15 and Lemma 14 directly.

Theorem 17 If a given metric is of Petrov II and if an NP tetrad for this metric is in the above canonical form then, after a gauge transformation $A \exp \left(\frac{i}{2} \alpha_{0}\right)=\left(\frac{\Psi_{4}}{\Psi_{2}}\right)^{\frac{1}{4}}$ to a new tetrad $\widehat{l}, \widehat{n}, \widehat{m}, \widehat{\bar{m}}$, all $(C) K V$ s can be found by applying $[\widehat{D}, £]=\frac{1}{2} \varphi \widehat{D}$ and its companion equations to the four coordinates $x^{i}$, and solving for $£_{\xi} x^{i}$ and the conformal function $\varphi$. Furthermore, (a) in the transformed tetrad, all properly conformally weighted combinations of the $N P$ scalars are Lie recurrent relative to each $(C) K V$, (b) in the transformed tetrad, all combinations of the NP scalars are Lie recurrent relative to each $K V$ and $H V$.

Corollary 18 The KVs only can be found by applying $[D, £]=0$ and its companion equations to the four coordinates $x^{i}$, and solving for $£_{\xi} x^{i}$. Furthermore, in the original tetrad, all combinations of NP scalars are Lie derived relative to each $K V$.

\footnotetext{
${ }^{5}$ It should be noted that the value of the constant for $\Psi_{4}$ differs for different references; we have chosen here, and in the other Petrov types to follow, the value used in the IC program[5.
} 
Note that the statement of this theorem is a bit more general than is required for a canonical tetrad of Petrov type II. In fact, it can apply to a tetrad with any gauge choice, not just for the canonical choice $\Psi_{4}=1$.

\section{Example 2.}

The Steele metric [16] considered by Koutras and Skea [7] is given by

$$
d s^{2}=r \ln r d u^{2}+2 r d x d u-r^{-\frac{1}{2}}\left(d r^{2}+d y^{2}\right) .
$$

In coordinates $(u, r, x, y)$ the tetrad vectors can be given by

$$
\begin{aligned}
l^{\alpha} & =\left(2 \sqrt{2} r^{\frac{1}{4}}, 0,-\sqrt{2} r^{\frac{1}{4}} \ln r, 0\right) \\
n^{\alpha} & =\left(0,0, \frac{1}{2 \sqrt{2}} r^{-\frac{5}{4}}, 0\right) \\
m^{\alpha} & =\left(0,-\frac{1}{\sqrt{2}} r^{\frac{1}{4}}, 0,-\frac{i}{\sqrt{2}} r^{\frac{1}{4}}\right) .
\end{aligned}
$$

$>$ From Eq.(35) we obtain

$$
\begin{array}{llll}
D u=2 \sqrt{2} r^{\frac{1}{4}} & D r=0 & D x=-\sqrt{2} r^{\frac{1}{4}} \ln r & D y=0 \\
\triangle u=0 & \triangle r=0 & \triangle x=\frac{1}{2 \sqrt{2}} r^{-\frac{5}{4}} & \triangle y=0 \\
\delta u=0 & \delta r=-\frac{1}{\sqrt{2}} r^{\frac{1}{4}} & \delta x=0 & \delta y=-\frac{i}{\sqrt{2}} r^{\frac{1}{4}} .
\end{array}
$$

The spin-coefficients are given by

$$
\begin{aligned}
& \nu=\rho=\sigma=\lambda=\mu=\varepsilon=\gamma=0 \\
& \tau=-\bar{\pi}=\frac{1}{2 \sqrt{2}} r^{-\frac{3}{4}}, \quad \kappa=2 \sqrt{2} r^{\frac{3}{4}}, \quad \alpha=-\frac{1}{2 \sqrt{2}} r^{-\frac{3}{4}}=2 \beta .
\end{aligned}
$$

and the nonzero components of the Riemann tensor by

$$
\Psi_{0}=1, \quad \Psi_{2}=\frac{1}{8} r^{-\frac{3}{2}}
$$

The metric is vacuum, of Petrov type II. The tetrad is canonical, with the gauge fixed by $\Psi_{0}=1$; therefore, we apply the gauge transformation 
$A \exp \left(\frac{i}{2} \alpha_{0}\right)=\left(\frac{\Psi_{2}}{\Psi_{0}}\right)^{\frac{1}{4}}$ and use Theorem 17 (slightly modified because it is $\Psi_{0}$, not $\Psi_{4}$, which equals one) to investigate all (C)KVs. Since the metric is vacuum we already know that no CKVs 15 exist; so we can apply Theorem 17 to find the HV using $\varphi=$ constant and statement (b).

Since $\Psi_{2}$ is Lie recurrent according to $£_{\xi} \Psi_{2}=-\varphi \Psi_{2}$, we can deduce that the coordinate $r$ is Lie recurrent according to $£_{\xi} r=\frac{2}{3} \varphi r$. Therefore, we first substitute $r$ into the commutators and find that the latter are identically satisfied.

Applying the NP-Lie commutator equations to the remaining coordinates in the transformed tetrad and solving these equations we find that

$$
\begin{aligned}
\varphi & =\text { constant }, \quad £_{\xi} u=\frac{1}{6} \varphi u+k, \\
£_{\xi} x & =\frac{1}{6} \varphi x-\frac{1}{3} \varphi u+b, \quad £_{\xi} y=\frac{2}{3} \varphi y+d
\end{aligned}
$$

where $k, b, d$ are integration constants. Therefore,

$$
\xi=\left(\frac{1}{6} \varphi u+k, \frac{2}{3} \varphi r, \frac{1}{6} \varphi x-\frac{1}{3} \varphi u+b, \frac{2}{3} \varphi y+d\right) .
$$

Putting, in turn, one of the integration constants equal to one and the remaining ones equal to zero we get the $\mathrm{HV}$

$$
\xi_{1}=u \frac{\partial}{\partial u}+4 r \frac{\partial}{\partial r}+(x-2 u) \frac{\partial}{\partial x}+4 y \frac{\partial}{\partial y}
$$

and the three KVs

$$
\xi_{2}=\frac{\partial}{\partial u}, \quad \xi_{3}=\frac{\partial}{\partial x}, \quad \xi_{4}=\frac{\partial}{\partial y} .
$$

Of course, the KV were obvious since the metric is independent of the $u, x$, and

$y$ - coordinates. There are no proper CKVs.

\subsection{PETROV TYPE III with a canonical tetrad}

In Petrov type III the canonical tetrad is fixed completely by the Weyl tensor: $\Psi_{3}=1$, all other $\Psi$ zero. Although this tetrad is preferred for any KVs that may exist in the spacetime, it is not necessarily preferred for an $\mathrm{HV}$ or 
for CKVs. Therefore, in Petrov type III, although a canonical tetrad is sufficient to investigate $\mathrm{KVs}$, in order to become a preferred tetrad with respect to a CKV, it will require a gauge transformation. Unfortunately, there are no other $\Psi$ components with which we can make a suitable gauge transformation (as was possible for Petrov II). So we may have to introduce explicitly an arbitrary gauge parameter which transforms the canonical tetrad to a preferred tetrad with respect to which we can then apply Theorem 15; we will find the value(s) for this parameter simultaneously while solving for the (C)KVs. In fact, because the condition $\Psi_{3}-\overline{\Psi_{3}}=0$ is properly conformally weighted, and it is only the condition $\Psi_{3}+\overline{\Psi_{3}}=2$ which is not properly conformally weighted, we will require only one arbitrary gauge parameter, namely a boost parameter $A$, to become a preferred tetrad with respect to a CKV.

Lemma 14 gives us the crucial recurrence results for the NP scalars associated with the preferred tetrad. Unfortunately, for the CKV case, the canonical tetrads are not preferred and so we cannot take the conclusion of Lemma 14 completely across to the NP scalars associated with the canonical tetrad; but we can use Lemma 14 for those NP scalars which are identical in both canonical and preferred tetrads, i.e., NP scalars which are independent of boost (i.e. those whose boost weight is zero), and which have proper conformal weight.

Theorem 19 If a given metric is of Petrov III and if an NP tetrad for this metric is in the above canonical form, then after a boost with a real parameter $A$ to a new tetrad $\widehat{l}, \widehat{n}, \widehat{m}, \widehat{\bar{m}}$, all $(C) K V$ s can be found by applying $[\widehat{D}, £]=\frac{1}{2} \varphi \widehat{D}$ and its companion equations to the four coordinates $x^{i}$, and solving for $£ x^{i}$, for $A$, and for the conformal function $\varphi$. Furthermore, (a) all boost- and properly conformally weighted combinations of the NP scalars are Lie recurrent relative to each $(C) K V$, (b) all boost-invariant combinations of the NP scalars are Lie recurrent relative to an $H V$ or a $K V$.

Corollary 20 The KVs only can be found by putting $A=1, \varphi=0$, and solving the commutator equations for $£_{\xi} x^{i}$. Furthermore, all combinations of the NP scalars are Lie derived relative to each $K V$. 


\subsection{PETROV TYPE D with a canonical tetrad}

In Petrov type D, a canonical tetrad is defined by $\Psi_{2} \neq 0$, all other $\Psi$ zero. It is clear that such a tetrad has preferred null directions, but all the gauge freedom remains arbitrary. Often this freedom is fixed explicitly in the process of determining the metric, either by reference to the Riemann tensor or the spin coefficients. However, it is important to realize that sometimes it is very difficult to fix the tetrad completely because of the complexity of the calculations, and also sometimes there is some unavoidable residual freedom due to isotropy which makes it impossible to fix the tetrad using the Riemann tensor and its derivatives. As in Type III, there are no more nonzero Weyl tensor components to enable us to fix the tetrad (but of course, in general, there could be Ricci tensor components, and derivatives of the Weyl and Ricci tetrad components).

If the gauge of the canonical tetrad has been fixed with respect to the Riemann tensor or its derivatives, then the tetrad is sufficient to investigate KVs. On the other hand, if the gauge has still some freedom, or is not fixed in a properly conformally weighted manner, or indeed if precisely how it is fixed is not immediately clear, we will still need to make a gauge transformation from the canonical to a preferred tetrad; arbitrary gauge parameters will have to be introduced which will be solved for, simultaneously with the (C)KVs.

For the CKV case, we cannot use Lemma 14 for all canonical NP scalars, but we can apply it to those scalars which are identical in both the canonical and preferred tetrad, i.e., scalars which are gauge invariant (whose spin and boost weights are both zero) and which have proper conformal weight.

Theorem 21 If a given metric is of Petrov D and if an NP tetrad for this metric is in the above canonical form then, after a gauge transformation with complex parameter $A \exp \left(\frac{i}{2} \alpha_{0}\right)$ to a new tetrad $\widehat{l}, \widehat{n}, \widehat{m}, \widehat{\bar{m}}$, all $(C) K V$ s can be found by applying $[\widehat{D}, £]=\frac{1}{2} \varphi \widehat{D}$, and its companion equations to the four coordinates $x^{i}$, and solving for $£_{\xi} x^{i}$, the unknown gauge parameters $A, \alpha_{0}$, and the conformal function $\varphi$. Furthermore, (a) all gauge invariant and properly conformally weighted combinations of the NP scalars are Lie recurrent relative to each $(C) K V$, (b) all gauge invariant combinations of the $N P$ scalars are Lie recurrent relative to an $H V$ or a $K V$.

It will be useful to have explicit examples of gauge invariant NP scalars, e.g., $\rho \mu, \pi \tau, \kappa \nu, \lambda \sigma, \Psi_{2}, \Psi_{0} \Psi_{4}, \Lambda, \Phi_{11}, \Phi_{00} \Phi_{22}, \Phi_{02} \Phi_{20}$ 
and gauge invariant and properly conformally weighted NP scalars, e.g., $(\rho-\bar{\rho})(\mu-\bar{\mu}),(\tau+\bar{\pi})(\bar{\tau}+\pi), \kappa \nu, \lambda \sigma, \Psi_{2}, \Psi_{0} \Psi_{4}$.

\section{Example 3.}

The Kimura metric [17] considered by Koutras and Skea|[7], given by

$$
d s^{2}=\frac{r^{2}}{b} d t^{2}-\frac{1}{r^{2} b^{2}} d r^{2}-r^{2} d \theta^{2}-r^{2} \sin ^{2} \theta d \phi^{2},
$$

is of Petrov type D with a non-zero energy momentum tensor. The metric is diagonal and, in coordinates $(t, r, \theta, \phi)$, we can readily construct the tetrad

$$
\begin{aligned}
l_{\alpha} & =\left(\frac{r}{\sqrt{2 b}},-\frac{1}{\sqrt{2} r b}, 0,0\right) \\
n_{\alpha} & =\left(\frac{r}{\sqrt{2 b}}, \frac{1}{\sqrt{2} r b}, 0,0\right) \\
m_{\alpha} & =\left(0,0,-\frac{r}{\sqrt{2}},-\frac{i r}{\sqrt{2}} \sin \theta\right),
\end{aligned}
$$

or, in contravariant form, 


$$
\begin{aligned}
l^{\alpha} & =\left(\frac{\sqrt{b}}{r \sqrt{2}}, \frac{r b}{\sqrt{2}}, 0,0\right) \\
n^{\alpha} & =\left(\frac{\sqrt{b}}{r \sqrt{2}},-\frac{r b}{\sqrt{2}}, 0,0\right) \\
m^{\alpha} & =\left(0,0, \frac{1}{r \sqrt{2}}, \frac{i}{r \sqrt{2} \sin \theta}\right) .
\end{aligned}
$$

It follows that

$$
\begin{array}{llll}
D t=\frac{\sqrt{b}}{r \sqrt{2}}, & D r=\frac{r b}{\sqrt{2}}, & D \theta=0, & D \phi=0 \\
\triangle t=\frac{\sqrt{b}}{r \sqrt{2}}, & \triangle r=-\frac{r b}{\sqrt{2}}, & \triangle \theta=0, & \triangle \phi=0 \\
\delta t=0, & \delta r=0, & \delta \theta=\frac{1}{r \sqrt{2}}, & \delta \phi=\frac{i}{r \sqrt{2} \sin \theta} .
\end{array}
$$

The NP spin coefficients are

$$
\begin{aligned}
& \kappa=\sigma=\lambda=\nu=\tau=\pi=0 \\
& \gamma=\varepsilon=\frac{b}{2 \sqrt{2}}, \quad \rho=\mu=-\frac{b}{\sqrt{2}}, \quad \beta=-\bar{\alpha}=\frac{\cot \theta}{2 \sqrt{2} r}
\end{aligned}
$$

and

$$
\Psi_{2}=-\frac{1}{6 r^{2}}, \quad \Phi_{11}=\frac{1}{4 r^{2}}, \quad \Lambda=-\frac{b^{2}}{2}+\frac{1}{12 r^{2}}
$$

are the only nonzero components of the Riemann tensor.

The tetrad is canonical, but it is not immediately obvious how, and to what extent, the gauge has been fixed; we cannot use the Ricci components and $\Psi_{2}$ to fix the gauge since all three components are invariant under gauge. So we apply Theorem 21 to investigate all (C)KVs.

We shall first investigate Lie recurrence relations for all (C)KV possibilities together, using (a). We note that $\Psi_{2}$ is gauge invariant and of conformal weight $w=-2$, and therefore $£_{\xi} \Psi_{2}=-\varphi \Psi_{2}$. Hence for the coordinate $r$ we have $£_{\xi} r=\frac{1}{2} \varphi r$. Although there is a second coordinate $\theta$ explicitly in the metric it does not occur in any gauge invariant properly conformally weighted scalars, and so we cannot use Theorem 21 to obtain a Lie recurrence relation for $\theta$. (On the other hand, $\theta$ does occur in the spin coefficients $\alpha$ and $\beta$, 
but these coefficients do not have appropriate behaviour for the theorem to apply.)

We therefore insert both a phase factor and a boost into our tetrad, so that Eq.(46) becomes

$$
\begin{array}{llll}
D t=\frac{A \sqrt{b}}{r \sqrt{2}}, & D r=\frac{A r b}{\sqrt{2}}, & D \theta=0, & D \phi=0 \\
\triangle t=\frac{\sqrt{b}}{A r \sqrt{2}}, & \triangle r=-\frac{r b}{A \sqrt{2}}, & \triangle \theta=0, & \triangle \phi=0 \\
\delta t=0, & \delta r=0, & \delta \theta=\frac{\exp \left(i \alpha_{0}\right)}{r \sqrt{2}}, & \delta \phi=\frac{i \exp \left(i \alpha_{0}\right)}{r \sqrt{2} \sin \theta} .
\end{array}
$$

Turning next to the NP-Lie commutator equations applied to the four coordinates we obtain, in addition to $£_{\xi} r=\frac{1}{2} \varphi r$, the following results.

$$
£_{\xi} t=h(r), \quad £_{\xi} \theta=f(\phi), \quad £_{\xi} \phi=g(\theta, \phi),
$$

where these four functions have to satisfy the equations

$$
\begin{aligned}
\frac{\partial g(\theta, \phi)}{\partial \theta} & =-\frac{1}{\sin \theta} £_{\xi} \alpha_{0} \\
\frac{d f(\phi)}{d \phi} & =\sin \theta £_{\xi} \alpha_{0} \\
\frac{\partial g(\theta, \phi)}{\partial \phi}+\cot \theta f(\phi) & =0 \\
\frac{1}{2} r \frac{\partial \varphi}{\partial r} & =\frac{\varphi(t, r, \theta, \phi)}{2} \\
A^{-1} £_{\xi} A & =r^{2} \sqrt{b} \frac{d h(r)}{d r} \\
A^{-1} £_{\xi} A & =\frac{1}{2 r \sqrt{b}} \frac{\partial \varphi}{\partial t}
\end{aligned}
$$

These equations are readily solved and we find that

$$
\begin{aligned}
\varphi & =\varphi(t, r)=r\left(2 l_{0} b t+l_{1}\right) \\
£_{\xi} r & =\frac{1}{2} r^{2}\left(2 l_{0} b t+l_{1}\right) \\
£_{\xi} t & =h(r)=-\frac{l_{0}}{r}+h_{0}, \\
£_{\xi} \theta & =f(\phi)=a_{0} \cos \phi+b_{0} \sin \phi \\
£_{\xi} \phi & =g(\theta, \phi)=c_{0}+\cot \theta\left(-a_{0} \sin \phi+b_{0} \cos \phi\right)
\end{aligned}
$$


as well as

$$
\begin{aligned}
A^{-1} £_{\xi} A & =l_{0} \sqrt{b} \\
£_{\xi} \alpha_{0} & =\frac{1}{\sin \theta}\left(-a_{0} \sin \phi+b_{0} \cos \phi\right),
\end{aligned}
$$

where $a_{0}, b_{0}, c_{0}, l_{0}, l_{1}, h_{0}$ are six arbitrary integration constants. Thus,

$$
\begin{aligned}
\xi & =\left(-\frac{l_{0}}{r}+h_{0}\right) \frac{\partial}{\partial t}+\frac{1}{2} r^{2}\left(2 l_{0} b t+l_{1}\right) \frac{\partial}{\partial r}+\left(a_{0} \cos \phi+b_{0} \sin \theta\right) \frac{\partial}{\partial \theta} \\
& +\left(c_{0}+\cot \theta\left(-a_{0} \sin \phi+b_{0} \cos \phi\right)\right) \frac{\partial}{\partial \phi} .
\end{aligned}
$$

Systematically putting all but one constant equal to zero we obtain the two CKVs

$$
\begin{array}{lll}
\xi_{(1)}=-\frac{1}{r} \frac{\partial}{\partial t}+r^{2} b t \frac{\partial}{\partial r} & \left(l_{0}=1,\right. & \varphi=2 r b t) \\
\xi_{(2)}=\frac{r^{2}}{2} \frac{\partial}{\partial r} & \left(l_{1}=1,\right. & \varphi=r)
\end{array}
$$

as well as the four KVs

$$
\begin{array}{ll}
\xi_{(3)}=\frac{\partial}{\partial t} & \left(h_{0}=1\right) \\
\xi_{(4)}=\frac{\partial}{\partial \phi} & \left(c_{0}=1\right) \\
\xi_{(5)}=\cos \phi \frac{\partial}{\partial \theta}-\cot \theta \sin \phi \frac{\partial}{\partial \phi} & \left(a_{0}=1\right) \\
\xi_{(6)}=\sin \phi \frac{\partial}{\partial \theta}+\cot \theta \cos \phi \frac{\partial}{\partial \phi}, & \left(b_{0}=1\right)
\end{array}
$$

two of which are obvious since the metric is independent of $t$ and $\phi$. There is no HV since $\varphi$ cannot be a nonzero constant. These symmetries have also been obtained by Koutras [18] and by O'Connor and Prince 19$]$.

In retrospect, we see that the phase part of the gauge cannot be fixed intrinsically; therefore, there is partial gauge isotropy. For, if it could be, this would imply that there is a common preferred phase for all four KV. That there is none follows from the second of Eqs. (53). We have,

$$
£_{\xi_{3}} \alpha_{0}=0=£_{\xi_{4}} \alpha_{0},
$$


implying that $\alpha_{0}=\alpha_{0}(r, \theta)$. But, by the same equation, to get a preferred phase for $\xi_{5}$ as well, we need

$$
£_{\xi_{5}} \alpha_{0}=-\frac{\sin \phi}{\sin \theta} \text {, i.e. } \cos \phi \frac{\partial \alpha_{0}(r, \theta)}{\partial \theta}=-\frac{\sin \phi}{\sin \theta} \text {. }
$$

This cannot be satisfied.

On the other hand, there is a boost $A$ so that the resultant boost part of the gauge is preferred for all KV. In fact, $A=1$ will do since the first of Eqs.(53) reduces to $£_{\xi} A=0$ for the four $\mathrm{KV}$ (and, therefore, there is no boost isotropy). That there is no common boost $A$ which will satisfy the first of Eq.(53) for all six (C)KV is readily verified.

This example illustrates how our approach deals with gauge isotropy. We do not need to know about possible gauge isotropy in advance; whatever the reason why a tetrad's gauge has not been fixed completely (or even if we are unsure whether it is fixed or not) we simply introduce the gauge parameters into the commutators, and the result of our calculation tells us whether our original gauge was completely preferred or not; and if the latter, it tells us what transformation takes the NP tetrad to a preferred gauge and what isotropy exists, if any.

\section{Example 4}

The Kerr-Newman metric 20 is of Petrov type D with an electromagnetic energy momentum tensor, and one standard version 21] is 


$$
\begin{aligned}
d s^{2} & =\left(1-\frac{2 m r-e^{2}}{\Sigma}\right) d u^{2}+2 d u d r+\frac{2 a \sin ^{2} \theta}{\Sigma}\left(2 m r-e^{2}\right) d u d \phi-2 a \sin ^{2} \theta d r d \phi \\
& -\Sigma d \theta^{2}-\sin ^{2} \theta\left(r^{2}+a^{2}+\frac{a^{2} \sin ^{2} \theta}{\Sigma}\left(2 m r-e^{2}\right)\right) d \phi^{2}
\end{aligned}
$$

where $\Sigma=r^{2}+a^{2} \cos ^{2} \theta$ and $\Delta=r^{2}+a^{2}+e^{2}-2 m r$. In the tetrad (with coordinates $(u, r, \theta, \phi))$

$$
\begin{aligned}
l^{\alpha} & =(0,1,0,0) \\
n^{\alpha} & =\left(\frac{r^{2}+a^{2}}{\Sigma},-\frac{\Delta}{2 \Sigma}, 0, \frac{a}{\Sigma}\right) \\
m^{\alpha} & =\frac{1}{\sqrt{2}(r+i a \cos \theta)}\left(i a \sin \theta, 0,1, \frac{i}{\sin \theta}\right)
\end{aligned}
$$

the only nonzero tetrad components of the Riemann tensor are

$$
\begin{aligned}
\Psi_{2} & =-\frac{m(r+i a \cos \theta)-e^{2}}{(r-i a \cos \theta)^{3}(r+i a \cos \theta)} \\
\Phi_{11} & =\frac{e^{2}}{2 \Sigma^{2}} .
\end{aligned}
$$

The tetrad is canonical, but it is not immediately clear how and to what extent the gauge has been fixed. We apply Theorem 21 to investigate all (C)KVs.

First, we consider how to obtain simple Lie recurrent relations for the coordinates $r, \theta$, using the second half of the theorem. Since we wish to consider all (C)KV possibilities together, using (a), we need to focus on NP scalars which are gauge invariant and properly conformally weighted: $\Psi_{2}$, $(\rho-\bar{\rho})(\mu-\bar{\mu}),(\tau+\bar{\pi})(\bar{\tau}+\pi)$. The simplest approach is to consider a combination which is gauge invariant, and also of conformal weight zero; e.g. $£_{\xi}\left((\rho-\bar{\rho})(\mu-\bar{\mu})(\tau+\bar{\pi})(\bar{\tau}+\pi) / \Psi_{2}\right)=0$. When we work this expression out explicitly we obtain, from its real and imaginary parts, two simultaneous homogenous equations in $£_{\xi} r$ and $£_{\xi} \theta$; since the determinant is non-zero, we conclude that $£_{\xi} r=0=£_{\xi} \theta$. Substituting this result into $£_{\xi} \Psi_{2}=-\varphi \Psi_{2}$ gives $\varphi=0$; this means that the Kerr-Newman metric does not permit an HV or CKVs. This example illustrates how we can obtain results on the 
non-existence of CKVs very efficiently, simply by testing the consistency of the NP scalars under conformal transformations.

To investigate the KVs only we could apply Theorem $21 \mathrm{~b}$ to $\Phi_{11}$ and $\rho$ (or to $\Psi_{2}$ ) and again obtain easily that $£_{\xi} r=0=£_{\xi} \theta$. It then remains to solve the NP-Lie commutator equations, with arbitrary gauge factor $A \exp \left(\frac{i}{2} \alpha_{0}\right)$, applied to the remaining two coordinates $u$ and $\phi$. It is easily seen that one possible solution for the gauge parameters is $A=1, \alpha_{0}=0$, showing that the original gauge is preferred relative to all KVs. The solution for the Lie derivatives of $u$ and $\phi$ is $£_{\xi} u=c_{1}, £_{\xi} \phi=c$, thereby yielding the two familiar $\mathrm{KVs}, \frac{\partial}{\partial t}$ and $\frac{\partial}{\partial \varphi}$.

\subsection{PETROV TYPE N with a canonical tetrad}

In Petrov type $\mathrm{N}$ a canonical tetrad is defined by $\Psi_{4}=1$, all other $\Psi$ zero.

Such a tetrad has one null direction preferred, namely the principal null direction, but the second null direction is not fixed. The gauge is fixed with respect to the Weyl tensor, but not in a proper conformally weighted manner. In practice, sometimes the null rotation freedom is fixed either by the spin coefficients or by the Riemann tensor; but there are some subclasses of metrics for which it is either difficult to determine a second null vector (because of complicated calculations), or even impossible to fix with respect to the Riemann tensor and its derivatives (because of null rotation isotropy).

If we can see that the null rotation freedom has been fixed intrinsically and in a proper conformally weighted manner, then the analysis is carried out as in Petrov type III with the introduction of only one arbitrary parameter - for a boost. But in all other cases, besides an arbitrary boost parameter $A$, it is necessary to introduce the arbitrary complex null rotation parameter $z$ explicitly into the commutators in order to obtain a transformation to a preferred tetrad.

In this case we can only use Lemma 14 for those scalars which are identical in both the canonical and preferred tetrad, i.e., scalars which are invariant under null rotations, which have proper conformal weight and which, in addition, are boost-invariant.

Theorem 22 If a given metric is of Petrov $N$ and if an NP tetrad for this metric is in the above canonical form then, after a null rotation with parameter $z$ and a boost with parameter $A$ to a new tetrad $\widehat{l}, \widehat{n}, \widehat{m}, \widehat{\bar{m}}$, all $(C) K V$ s

can be found by applying $[\widehat{D}, £]=\frac{1}{2} \varphi \widehat{D}$ and its companion equations to the 
four coordinates $x^{i}$, and solving for $£_{\xi} x^{i}$, the unknown gauge parameter $A$, and the unknown null rotation parameter $z$ and the conformal function $\varphi$. Furthermore, (a) all null rotation invariant, boost-invariant and proper conformally weighted combinations of the NP scalars are Lie recurrent relative to each $(C) K V$, (b) all null rotation invariant and boost-invariant weighted combinations of the NP scalars are Lie recurrent relative to an $H V$ and any $K V$.

Corollary 23 The KVs only can be found by putting $A=1, \varphi=0$, and solving the commutator equations for $£_{\xi} x^{i}$ and the unknown null rotation parameter $z$. Furthermore, all null rotation invariant combinations of the $N P$ scalars are Lie derived relative to each $K V$.

\subsection{PETROV TYPE O}

For Petrov type 0 spacetimes another approach has to be used - usually using the Ricci tensor to fix part of the tetrad - and although we can still handle those cases with the general principles described in this paper, we will postpone such considerations to a subsequent paper. 


\subsection{ALL TYPES}

We emphasise that in practical calculations involving NP tetrads, using canonical tetrads is not the only way in which NP tetrads can be chosen. For instance, it is useful in some circumstances for the NP tetrad to be partly fixed with respect to the Weyl tensor and partly with respect to the Ricci tensor. Also, in investigations concerning the IC of metrics, canonical tetrads are obtained which have more complicated relationships (involving derivatives of the Riemann tensor) with the Weyl and Ricci tensors. Therefore, in Petrov types III, D, N where we have introduced arbitrary parameters, an alternative procedure may be to first of all find an explicit suitable gauge transformation or null rotation, in order to avoid using some of the arbitrary parameters - in a manner analogous to the one we have used our discussion of Petrov type II. But this will require a deeper analysis of the tetrad, an analysis which involves determining how the tensor is fixed with respect to derivatives of the Riemann tensor, as in the IC. However, we point out that although we should always be able to eliminate the gauge parameter for Petrov type III, we cannot always eliminate the parameters in Petrov types $\mathrm{D}$ and $\mathrm{N}$ because of isotropy.

Our general approach outlined in the previous subsections is designed to include as well tetrads other than the ones discussed above, and we shall investigate such situations in a subsequent paper. However, using canonical tetrads is a familiar approach, and in this paper we wished to illustrate the efficiency of our method with familiar examples.

\section{Conclusion}

The main thrust of this paper was to present a very efficient, alternative, approach to the problem of finding (conformal) Killing vectors, i.e. KVs, $\mathrm{HV}$, or CKVs. Although our examples have dealt with finding such vectors for a given metric, our technique works equally well when solving Einstein's equations for a spacetime with such symmetries.

This technique consists of the replacement of the usual (conformal) Killing equations with sixteen equations involving commutators of the Lie derivative with the four NP differential operators applied to the four coordinates. These operators must correspond to a tetrad which is preferred relative to the (C)KV to be found. This notion was defined for null directions in an earlier 
paper|2 and extended to gauge in this one. In practice, we usually need a tetrad which is preferred relative to all $\mathrm{KVs}$ and/or $\mathrm{HV}$ and/or CKVs of the metric. To cover all possible $(\mathrm{C}) \mathrm{KVs}$ it is best to find null directions which are intrinsically defined by the Weyl tensor alone (in a proper conformally weighted manner). As for gauge, if we want to find only all KVs it suffices to choose one which has been defined intrinsically. To get CKVs as well, we need to look for a gauge which has been defined not only intrinsically but in a proper conformal manner as well.

In such a preferred gauge the calculations for the $(\mathrm{C}) \mathrm{KVs}$ become relatively simple, especially if as many coordinates as possible are chosen intrinsically as well. In case of isotropy, or if ignorant of a tetrad which is preferred relative to all the $(\mathrm{C}) \mathrm{KVs}$ we want to find, we must put an arbitrary null rotation parameter and/or an arbitrary gauge factor into the tetrad used. Then the calculations become a bit longer. The NP-Lie commutator equations must now be solved for the Lie derivative of such a factor as well as for the Lie derivatives of the coordinates. Clearly, in the interest of simplicity of calculations, we should choose as much of the tetrad to be preferred relative to all vectors of interest, so as to minimize the use of null rotation and/or boost-rotation parameters. This can often be done in the manner described and illustrated in section 4 . The choice depends on whether we're looking only for KVs, for an $\mathrm{HV}$ and KVs, or for CKVs as well. Further simplification is achieved by choosing as many coordinates as possible in an intrinsic fashion. All the KVs, any HV, and all the CKVs are then readily obtained.

Our results for (C)KVs apply to proper conformal Killing vectors, homothetic vectors and Killing vectors. It might be thought superfluous for us to give specific results valid only for KVs or an HV. However, as noted in the introduction, there are some general theorems which, in certain circumstances, rule out the existence for CKVs. In such circumstances it is more efficient to use the results which are valid only for $\mathrm{KVs}$ and/or an $\mathrm{HV}$.

The examples which we have used to illustrate our method are comparatively simple, being metrics quoted with tetrads in canonical form. However, our method is applicable, and efficient, for other types of tetrad. In particular, our method can be used to extend the IC program by determining explicitly the form of those KVs and HVs whose existence can be concluded from the existing IC program. In addition, our results will enable the IC program to deal with HVs and CKVs. These applications will be published in a subsequent paper. 


\section{ACKNOWLEDGEMENTS}

One author (BE) gratefully acknowledges the financial support by the Natural Science Research Council of Sweden, the travel support from the Magnusson Fund, Swedish Royal Academy, and the hospitality of the Department of Mathematical Sciences, University of Alberta, Canada. The other author (GL) would like to thank the Natural Sciences and Engineering Research Council of Canada for its ongoing financial support.

\section{References}

[1] Edgar S.B. and Ludwig G. (2000). Gen. Rel. Grav. 32, 637-671.

[2] Ludwig G. and Edgar S. B. (2000). Class. Quantum Grav. 17, 1683-1705.

[3] Geroch R., Held A., and Penrose R. (1973). J. Math. Phys. 14, 874-881.

[4] Newman E.T. and Penrose R. (1962). J. Math. Phys. 3, 566-578.

[5] Karlhede A. (1980). Gen. Rel. Grav. 12, 693-707.

[6] Karlhede A. and MacCallum M.A.H.(1982). Gen. Rel. Grav. 14, 673-682.

[7] Koutras A. and Skea J.E.F. (1998). Computer Physics Communications 115, 350-362.

[8] Hall G.S. (2000). Gen. Rel. Grav. 32, 933-941.

[9] Hall G.S. (2000). Class. Quantum Grav. 17, 3073-3076.

[10] Jerie M., O’Connor, J.E.R. and Prince G.E. (1999). Class. Quantum Grav. 16, 2885-2887.

[11] Kolassis Ch. and Ludwig G. (1993). Gen. Rel. Grav. 25, 625-651.

[12] Ludwig G. (1988). Int. J. Theor. Phys. 27, 315-333.

[13] Machado Ramos M.P. and Vickers J.A. (1996). Class. Quantum Grav. 13, 1579-1587.

[14] Bell P. and Szekeres P. (1972). Int. J. Theor. Phys. 6, 111-121. 
[15] Hall G.S. and Keane A.J. (2000). Class. Quantum Grav. 17, 1571-1573.

[16] Steele J.D. (1991). Gen. Rel. Grav. 23, 811-825.

[17] Kimura M. (1976). Tensor 30, 27-43.

[18] Koutras A. (1992). Class. Quantum Grav. 9, 1573-1580.

[19] O’Connor, J.E.R. and Prince G.E. (1998). Gen. Rel. Grav. 30, 69-82.

[20] Newman E.T. et al. (1965). J. Math. Phys. 6, 918-919.

[21] Bose S.K. (1975). J. Math. Phys. 16, 772-775. 